GRADIATION\&APPLICATIONS

ISSN 2466-4294 (online) | rad-journal.org

Vol. 3 | Issue 1 | pp. 13-17, 2018

doi: 10.21175/RadJ.2018.01.003

Original research paper

\title{
INFLUENCE OF LOW IODINE-131 DOSES ON SUSCEPTIBILITY TO IONIZING RADIATION AND BIOMARKERS OF HEALTH RISK*
}

\author{
Antonina Cebulska-Wasilewska ${ }^{1,3^{* *}}$, Mateusz Krzysiek ${ }^{2}$, \\ Grażyna Krajewska', Artur Stępieńn ${ }^{3}$, Paweł Krajewski ${ }^{1}$
}

\author{
${ }^{1}$ Central Laboratory for Radiological Protection, Warsaw, Poland \\ ${ }^{2}$ Institute of Nuclear Physics, Polish Academy of Sciences, Krakow, Poland \\ ${ }^{3}$ Central Center for Radiation Emergency, $5^{\text {th }}$ Military Hospital \& Public Polyclinic, Krakow, Poland
}

\begin{abstract}
An emergence of ${ }^{131}$ in ambient air might be one of the first signs of a mishap. Even though precautions are clearly established, nuclear power plant accidents or any radioactive threat might occur. Iodine in the human body preferentially concentrates in the thyroid, so ${ }^{131} I$ is frequently used in nuclear medicine to diagnose or cure problems with it. We have previously reported strong variability in health risk biomarkers detected in lymphocytes of patients after diagnostic and therapeutic I-131 applications. Now, we report cellular responses to a challenging high dose of X-rays applied in vitro, as well as the DNA repair capacity examined in lymphocytes isolated from whole blood samples collected from 41 subjects exposed to the diagnostic ${ }^{131}$ I dose, and 30 persons who were unexposed. The aim of the study was to find out if individual susceptibilities to ionizing radiation (IR), defined by molecular and cellular repair capacities, of persons diagnosed with very low I-131 doses are different from those observed in an unexposed control group, and how confounding factors - age, gender, family vulnerability to cancer, and polymorphism in genes associated with repair - affect it. The DNA repair competence assay was applied using the Comet method. The RDT-DNA (residual DNA damage, percentage of unrepaired DNA during post irradiation incubation) was used as a biomarker of fast DNA repair on a molecular level and was compared to. SCE levels (sister chromatid exchanges) measured on a cellular level as biomarker associated with cellular repair via homologous recombination. On average, lymphocytes of the subgroup diagnosed by ${ }^{131}$ I expressed a statistically significant increase in repair efficiency of DNA damage induced by a challenging dose as compared to the average value from the respective unexposed control group. That increase was followed by a strong decrease in the percentage of cells with a significantly elevated number of SCE and frequency of cells with significantly elevated numbers of SCE (HFChigh frequency cells). The observed increase of DNA repair efficiency also corresponded to previously reported significant decreases of chromosome aberrations levels, and to MN frequencies known as biomarkers of health risk. Nevertheless, all investigated biomarkers were characterized by high variability between individual responses. Obtained results show a slight dependence on gender and the family's predisposition to cancer, and a significant dependence on polymorphism in the XRCC1(194), XRCC1(399), and XRCC13(241) genes involved in DNA repair process. While it is necessary to increase the number of polymorphism studies, we propose a model of short-term biomarker battery applicable for triage and prediction of the health risk from any ionizing radiation exposure.
\end{abstract}

Key words: Biomarkers, DNA repair competence assay, HFC, Iodine-131, individual susceptibility, SCGE, SCE, XRCC polymorphism

\section{INTRODUCTION}

The challenge of environmental studies valuable for molecular epidemiology is to advance and improve processes for assessing risk to human health from exposure to all types of genotoxic agents, including ionizing radiation. It is well-determined that exposure to low doses of ionizing radiation (IR) is unavoidable due to its presence in the natural environment. Nevertheless, high exposures to IR can result from nuclear plant accidents, cancer treatment, or even acts of terrorism, non-specific contamination, industrial discharges. Occupational environment can also results in exposure to other types of genotoxic agents. Therefore, the population can be potentially exposed to a complex mixture of IR and various genotoxic agents that could cause harmful effects within days, weeks, or years. While the use of radiation around the world offers a wide range of benefits in medicine, energy production, industry and research, safety precautions are essential to understand and moderate unnecessary exposure of people. The emergence of the ${ }^{131} \mathrm{I}$ in ambient air might be one of the first signs of nuclear mishap. Even though precautions are clearly established, nuclear power plant accidents or any

\footnotetext{
* The paper was presented at the Fifth International Conference on Radiation and Applications in Various Fields of Research (RAD 2017), Budva, Montenegro, 2017.

b7wasile@cyf-kr.edu.pl
} 
A. Cebulska-Wasilewska et al., Influence of low iodine-131 doses..., Rad. Applic., 2018, 3, 1, 13-17

radioactive threat might still happen. Iodine in the human body preferentially concentrates in the thyroid, so ${ }^{131} \mathrm{I}$ is frequently used in nuclear medicine to improve health conditions by early disease diagnosis and restore health by slowing down an overactive thyroid or even killing cancer cells. Nevertheless, external or internal exposures to radioiodine can also lead to molecular and cellular damage not only in thyroid cells but also in other tissues, including peripheral blood lymphocytes. It can also increase the occupational risk. For that reason, a reliable estimate and prediction of normal tissue/cell- response to ionizing radiation and consequent health risk from exposure would be very useful in occupational radiation protection, radiotherapy and the population's risk estimation. We have earlier reported a strong variability in health risk biomarkers detected in lymphocytes of patients after diagnostic and therapeutic I-131 applications [1]-[3]. The aim of this study was to find out if individual susceptibilities to IR, defined by molecular and cellular repair capacities investigated in lymphocytes exposed in vitro to a high dose of X-rays differed between persons diagnosed with very low I-131 doses from an unexposed control group. We wanted also to find out if confounding factors - family vulnerability to cancer and polymorphism in associated to repair genes - can affect vulnerability to IR and DNA repair.

\section{MATERIAL AND METHODS}

The general study group [1] consisted of 41 subjects diagnostically exposed to low doses of I-131 (in the range of $\left.1.85 \sim 4.45 \mathrm{MBq}, \mathrm{A}_{\mathrm{Av}}=2.96 \pm 0.82 \mathrm{MBq}\right)$ and 37 patients who had returned to the polyclinic for hyperthyroidism treatment with I-131 (in the range of $300 \sim 650 \mathrm{MBq}, \mathrm{A}_{\mathrm{Av}}=497.3 \pm 88.1 \mathrm{MBq}$ ) [1]-[3]. All subjects voluntarily gave blood samples and responded to a questionnaire describing their life-styles, hobbies, and health conditions, as well as their occupational history and hazards. Blood samples from thyroid patients were collected after scintigraphy performed with a very low dose of I-131, and just before application of the therapeutic dose [1]. A control group consisted of 30 unexposed volunteers, free of thyroid diseases and considering themselves healthy. Lymphocytes from group of 60 subjects, randomly selected from the unexposed control group and the group of hyperthyroidism patients, were chosen to find out how the polymorphism of genes encoding proteins involved in radiation-induced-DNA-damage repair influences individual vulnerability to IR. Three types of XRCC genes (from the X-ray cross complementary group) were chosen for this study: XRCC1(194) and XRCC1(399), involved in DNA single strand brakes and base excision repair (BER) processes; and $\mathrm{XRCC}_{3}$ (241), involved in the repair of DNA double-strand brakes by homologous recombination (HR). The analysis of polymorphisms spectrum was performed by the Oncogene, a professional clinical firm [4]. To study vulnerability to IR at the cellular level, part of the whole blood samples were irradiated in vitro in heparinized tubes with a dose of $2 \mathrm{~Gy}$ and culture procedures were performed following appropriate
procedures.All the details about the biomarker methods applied for this study - the DNA repair competence with SCGE assay (known also as a Comet assay), and the cytogenetic study for sister chromatid exchanges (SCE) and high frequency cells (HFC) - are well known and were described in details elsewhere [3].[5],[6]. Statistical analysis was performed with the use of ANOVA analysis of variance from SPSS, statistical program for social science.

\section{RESULTS}

Table 1 presents comparisons between mean values of molecular biomarkers detected for investigated groups of subjects selected for polymorphism study the unexposed control (CG) and the one diagnostically exposed to low I-131 (DG).

Table 1. Comparison between mean values of molecular biomarkers detected for investigated groups: control (CG) and diagnostically exposed (DG) to a low I-131 dose.

\begin{tabular}{|c|c|c|c|}
\hline & T-DNA & T-DNAGy $_{\text {G }}$ & $\begin{array}{c}\text { RD }_{\text {T-DNA }} \\
{[\%]}\end{array}$ \\
\hline CG & $\mathbf{6 . 8}$ & $\mathbf{1 5 . 1}$ & $\mathbf{3 8 . 3}$ \\
\hline \pm SD & 2.5 & 3.1 & 22.9 \\
\hline DG & $\mathbf{5 . 7}$ & $\mathbf{1 5 . 9}$ & $\mathbf{2 2 . 1}^{*}$ \\
\hline \pm SD & 1.5 & 2.9 & 11.5 \\
\hline
\end{tabular}

T-DNA ${ }_{0}$ - DNA damage detected by SCGE assay in lymphocytes not irradiated in vitro. T-DNA $\mathrm{Gy}_{\mathrm{G}}$ - DNA damage assessed by SCGE assay and detected in irradiated lymphocytes immediately after X-ray exposure. RD T-DNA residual DNA damage expressed as a percentage of DNA damage unrepaired during post-irradiation incubation. ${ }^{*}$ significance $<0.05$

Table 2. Comparison between mean values of the cytogenetic biomarkers detected for investigated groups: (CG) and diagnostically exposed to a low dose of (DG) I-131.

\begin{tabular}{|c|c|c|c|}
\hline & SCE & SCE $_{\max }$ & $\begin{array}{c}\text { HFC } \\
{[\%]}\end{array}$ \\
\hline CG & $\mathbf{6 . 3}$ & $\mathbf{1 3 . 1}$ & $\mathbf{3 2 . 4}$ \\
\hline \pm SD & 1.4 & 2.0 & 18.5 \\
\hline DG & $\mathbf{5 . 7}$ & $\mathbf{1 2 . 0}$ & $\mathbf{2 3 . 0}$ \\
\hline \pm SD & 1.6 & 2.7 & 17.5 \\
\hline
\end{tabular}

SCE - sister chromatid exchanges per cell; SCE $\max$ - average from the maximal values of SCE detected for subjects in the studied group; HFC - percentage of cells with significantly elevated amount of SCE.

A statistically insignificant decrease from the level detected in the control group is observed in the diagnostic group in not irradiated lymphocytes (T-DNAo). No difference between CG and DG is observed in the level of DNA damage detected immediately after irradiation in vitro with challenging dose. In the group exposed to diagnostic I-131 (low dose), a significant decrease from the mean levels detected in the control group is observed for $\mathrm{RD}_{\text {T-DNA }}$ (the percentage of DNA damage (Residual) unrepaired during post-irradiation incubation), that confirms more efficient fast DNA repair. Table 2 presents comparisons between mean values of cytogenetic biomarkers detected for the investigated groups - the 
unexposed control (CG) group and the one exposed diagnostically to low I-131 (DG).

Although mean values of the cytogenetic biomarkers detected in unexposed control (CG) group are visibly higher than those detected for exposed diagnostically group (DG) though differences are not significant. Table 3 presents comparisons between the mean values of cytogenetic biomarkers detected for the investigated groups, (CG) and (DG), in lymphocytes irradiated in vitro by a challengingly high $(2 \mathrm{~Gy})$ dose of X-rays.

Table 3. Comparison between mean values of the cytogenetic biomarkers detected for the investigated groups, (CG) and (CG), in lymphocytes irradiated in vitro by a challengingly high X-ray (2Gy) dose

\begin{tabular}{|c|c|c|c|}
\hline & SCE $_{G y}$ & SCE $_{\operatorname{maxGy}}$ & $\begin{array}{c}\mathrm{HFC}_{\mathrm{Gy}} \\
{[\%]}\end{array}$ \\
\hline CG & $\mathbf{6 . 7}$ & $\mathbf{1 2 . 6}$ & $\mathbf{3 3 . 5}$ \\
\hline \pm SD & 1.7 & 2.1 & 19.8 \\
\hline DG & $\mathbf{6 . 0}$ & $\mathbf{1 2 . 6}$ & $\mathbf{2 6 . 7}$ \\
\hline \pm SD & 1.6 & 3.3 & 17.6 \\
\hline
\end{tabular}

Abbreviations as shown in Table 2.

No difference between CG and DG groups is observed in the level of $\mathrm{SCE}_{\mathrm{Gy}}$ and $\mathrm{SCE}_{\operatorname{maxGy}}$ detected in cells after in vitro irradiation with a challenging dose. Although $\mathrm{HFC}_{\mathrm{Gy}}$ for the DG group is visibly lower than that detected in the control group, again the difference is statistically is insignificant. However, it is worth mentioning that a strong variability is observed in molecular and cytogenetic biomarkers detected in lymphocytes of both investigated groups (control group and I-131 diagnosed patients). Results presented in Figure 1 show how polymorphism, in genes encoding proteins involved in fast DNA repair, is influencing responses of investigated biomarkers.

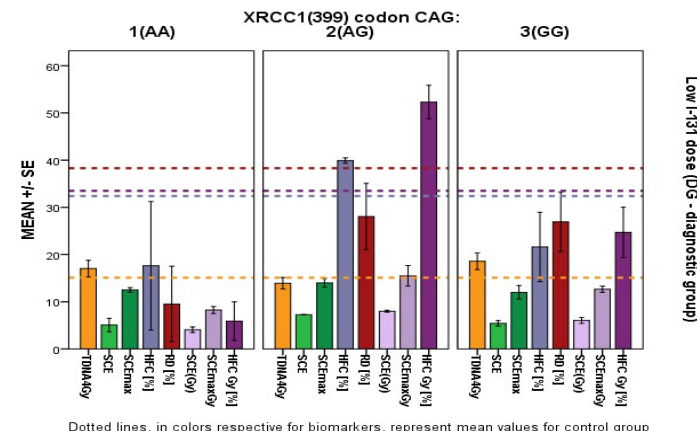

Figure 1. Differences between molecular and cytogenetic biomarkers, involved in various mechanisms (BEIR and HR) of DNA repair, detected in subgroups stratified according to polymorphic form of gene XRCC1(399) codon CAG.

Subjects characterized by heterozygous (AG) form of the XRCC1(399) gene codon CAG, are expressing the lowest efficiency of fast DNA repair (the highest RDTDNA level) and highest HFC levels (percentage of cells with significantly elevated numbers of SCE which is involved in homologous recombination.
Results presented in Figure 2 show how polymorphism in gene $\mathrm{XRCC}_{3}(241)$ codon ACG, encoding proteins involved in the DNA repair via homologous recombination, is influencing responses of investigated biomarkers.

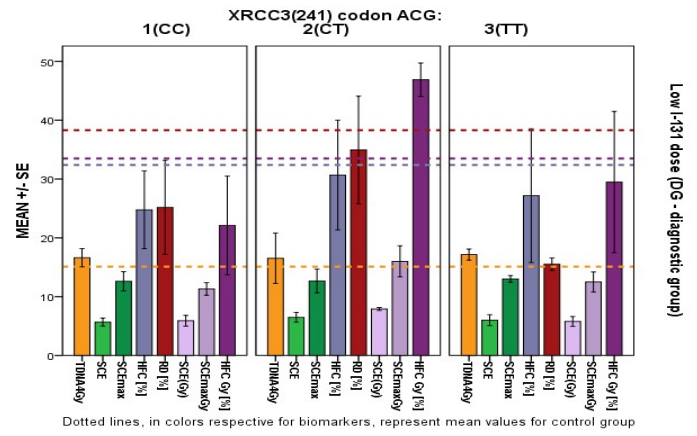

Figure 2. Differences between molecular and cytogenetic biomarkers, involved in various mechanisms of DNA repair, detected in subgroups stratified according to polymorphic form of gene $\mathrm{XRCC}_{3}(241)$ codon ACG.

Similarly, subjects characterized by heterozygous (CT) form of the gene $\mathrm{XRCC}_{3}$ (241) codon CAG, are expressing the lowest efficiency of the fast DNA repair (the highest RDT-DNA) and the highest HFC levels (percentage of cells with significantly elevated numbers of SCE which is involved in homologous recombination. Moreover, (in both Fig.1 and Fig.2) almost no differences between polymorphic subgroups are seen in susceptibility to IR, that is demonstrated by $\mathrm{T}^{-D_{N A}} \mathrm{~Gy}_{\mathrm{g}}$ levels, neither in sister chromatid exchanges

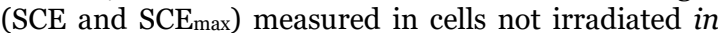
vitro with high challenging $\mathrm{X}$-ray dose.

In this study, we have investigated various (molecular and cytogenetic) biomarkers in the same pool of cells. According to the old molecular theory of radiation biology by K.H. Chadwick and H.P. Leenhouts [7], if the DNA repair process is not affected, correlation between various biomarkers in such cases should be strong and linear. Figure 3 shows a correlation between molecular ( $\left.\mathrm{RD}_{\mathrm{T}-\mathrm{DNA}}\right)$ and cytogenetic biomarkers ( HFC $_{\text {Gy }}$ ) for CG and DG after stratification into polymorphic subgroups (1 homozygous $\mathrm{C} / \mathrm{C}, 2-$ heterozygous $\mathrm{C} / \mathrm{T}, 3-$ homozygous $\mathrm{T} / \mathrm{T}$ ) of the ACG codon in the $\mathrm{XRCC}_{3}$ (241) gene.

Family predisposition to cancer is also marked. Red circles show subjects with a positive response regarding the Cancer in Immediate Family (CiF) reports. Frequency of subjects in the subgroup with polymorphic genes is different for the CG and DG groups. The dispersion from the straight line is huge and higher for $\mathrm{C} / \mathrm{T}$ heterozygous subgroups and mutant T/T. Figure 4 shows the correlation between molecular (RDT-DNA) and cytogenetic biomarkers $\left(\mathrm{HFC}_{\mathrm{Gy}}\right)$ for CG and DG groups after stratification to polymorphic subgroups of the XRCC1(399) gene (1 homozygous A/A, 2 - heterozygous $\mathrm{A} / \mathrm{G}$ and $3-$ homozygous $\mathrm{G} / \mathrm{G})$. 


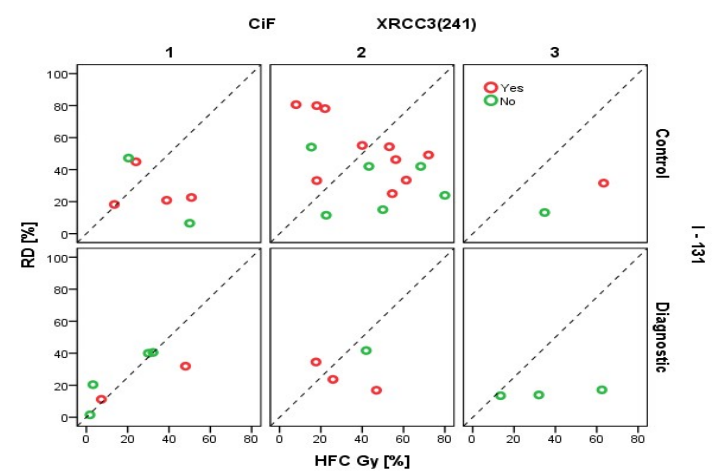

Figure 3. Correlation between molecular (RDT-DNA) and cytogenetic biomarker $\left(\mathrm{HFC}_{\mathrm{Gy}}\right.$ ) for CG and DG after stratification to polymorphic subgroups for the ACG codon in the $\mathrm{XRCC}_{3}$ (241) gene. Red circles show subjects with Cancer

in Immediate Family $(\mathrm{CiF})$ positive reports, green circles indicate no cancer reports.

A similar discrepancy is observed in the control CG group for the polymorphic subgroup in codon CAG of the XRCC1(399) gene involved in the DNA single strand breaks repair; however, the spectrum in DG is different. It seems that subjects with homozygous G/G of this gene showed a good correlation between molecular and cytogenetic damage. Red circles again show subjects with a positive response regarding the Cancer in Immediate Family (CiF) reports. In both figures shown, the influence of the CiF factor on those CG and TG seems to be less important.

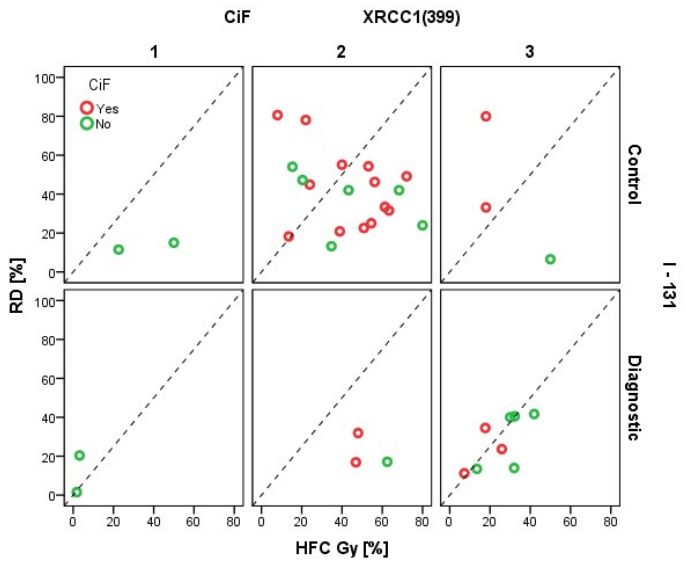

Figure 4. Correlation between molecular $\left(\mathrm{RD}_{\mathrm{T}-\mathrm{DNA}}\right)$ and cytogenetic biomarkers $\left(\mathrm{HFC}_{\mathrm{Gy}}\right.$ ) for CG and DG after stratification to polymorphic subgroups for the CAG codon (1 - homozygous A/A, 2 - heterozygous A/G and homozygous G/G) codon ACG in the XRCC1 (399) gene. Red circles show subjects with positive Cancer in Immediate Family (CiF) reports, green circles indicate no cancer reports.

The obtained results show that high variability between individuals in the response and vulnerability to IR is strongly dependent on polymorphism in genes involved in DNA repair.

\section{CONCLUSION}

Radiation sensitivity is one of the main factors that differentiate individuals in terms of the effectiveness of therapeutic procedures, or its side effects, considering the fact how well the damage incurred on the cells is repaired. Individual variation in response to exogenous agents is expected as the genetic background, metabolic rate and the DNA repair capacity influence such responses. On average, lymphocytes of the ${ }^{131} \mathrm{I}$ diagnosed subgroup expressed a statistically significant increase in the repair efficiency of DNA damage induced by a challenging dose as compared to the average value from respective unexposed control group. That increase was followed by a strong, although insignificant decrease in the percentage of cells with a significantly elevated number of SCE (HFC high frequency cells). The observed increase of DNA repair efficiency also corresponded to the significant decrease to $\mathrm{CA}$ levels, and to reported earlier $\mathrm{MN}$ frequencies [2]. However, all investigated biomarkers were characterized by high variability between individual responses. The obtained results show a slight dependence on the family's predisposition to cancer, and a significant dependence on polymorphism in the XRCC1(399), XRCC1(399), and XRCC13(241) genes. While additional polymorphism studies are necessary, the model of biomarker battery, which proposes a combination of short-term biomarkers with molecular genetics as a more reliable risk predictor, is applicable for triage and health-risk estimation procedural action after anyexposure to high IR dose, including cancer treatment. Recent conclusive results show that the level of detected cytogenetic damage and reliability of the estimated biomarkers of secondary cancer risk might be affected not only by the range of the administered dose and the length of time between iodine administration and sampling, but also mainly by the alteration of DNA repair process due to genetic predisposition, occupational hazard, and the lifestyle of the exposed individuals. The extension and deepening of studies of those samples (i.e., of the polymorphisms spectrum of genes involved in the repair mechanism of the DNA damage induced by radiation) can result in a combination of fast and inexpensive biomarkers that might give an even more accurate prediction of individual radio-sensitivity, which could also be important for clinical applications. More studies might also help to fulfill gaps in this knowledge and support a better understanding of the mechanism behind observed variability in responses to radiation.

Acknowledgement: Research partially supported by: MNiSZW 2520/B/PO1/201O/39, nr.N4O4 252039 and "Technologies supporting the development of safe nuclear power" - (NCBiR). Research Task "Development of methods to assure nuclear safety and radiation protection for current and future needs of nuclear power plants", contract No.SP/J/6/143339/11 and IAEA's Coordinated Research Project (CRP) No.17101. "Strengthening of Biological dosimetry". Assistance in samplings and coding by Dr. J.Pawlus and Dr. Z.Drag is greatly appreciated. 
A. Cebulska-Wasilewska et al., Influence of low iodine-131 doses..., Rad. Applic., 2018, 3, 1, 13-17

\section{REFERENCES}

1. J. Gasiorkiewicz et al., "Studies on response to the challenging dose of $x$-rays in lymphocytes of patients undergoing diagnosis and treatment with Iodine-131," in NATO Science for Peace and Security Series E: Human and Societal Dynamics: Rapid Diagnosis in Populations at Risk from Radiation and Chemicals, vol. 73, A. Cebulska-Wasilewska, A. N. Osipov, F. Darroudi, Eds., Amsterdam, Netherlands: IOS Press, 2010.

2. A. Cebulska-Wasilewska, J. Miszczyk, Z. Drag, J. K. Kim, "Health Risk Impact due to Exposure to ${ }^{131}$ with Low and High Doses Evaluated with Micronucleus Assay," J. Korean Radiat. Ind., vol. 5, no. 4. pp. 331 335, 2011.

3. A. Cebulska-Wasilewska, M. Krzysiek G. Krajewska, A. Stępień, P.Krajewski, "Retrospective biological dosimetry at low and high doses of radiation and radioiodine impact on individual susceptibility to ionizing radiation, Genome Integrity, vol. 8, no. 1, pp. 2 - 12, Jan. 2017.

DOI: $10.4103 / 2041-9414.198906$

PMid: 28250909

PMCid: PMC5320787
4. Oncogene Diagnostics, Oncogene Diagnostics, Krakow, Poland.

Retrieved from: http://www.oncogene.pl

Retrieved on: Jan. 17, 2017

5. A. Cebulska-Wasilewska, "Response to challenging Dose of X-rays as a Predictive Assay for Molecular Epidemiology," Mutat. Res. Rev. Mutat. Res., vol. 544 no. 2-3, pp. 289 - 297, Nov. 2003. DOI: 10.1016/j.mrrev.2003.07.003

6. A. Cebulska-Wasilewska, J. Rachtan, Z. Rudek, Z. Drag, "Cytogenetic damage detected in lymphocytes of donors from Małopolska region in Poland and cancer incidence in the follow-up studies," in Environmental Health in Central and Eastern Europe, K. C. Donnelly et al., Eds. Berlin, Germany: Springer, 2006, ch. 7, pp. $53-64$. DOI: $10.1007 / 1-4020-4845-9$

7. K. H. Chadwick, H. P. Leenhouts, The Molecular Theory of Radiation Biology, Berlin, Germany: Springer-Verlag, 1981.

DOI: $10.1007 / 978-3-642-81519-5$ 